\title{
Head Injury Prediction Methods Based on 6 Degree of Freedom Head Acceleration Measurements during Impact
}

\author{
Hideyuki Kimpara $^{1)}$ Yuko Nakahira $^{2)}$ Masami Iwamoto $^{3)}$ \\ Steve Rowson ${ }^{4)}$ Stefan Duma ${ }^{5)}$ \\ 1)-3) Toyota Central R\&D Labs., Inc. \\ 41-1 Yokomichi, Nagakute, Aichi 480-1192, Japan (E-mail: h-kimpara@mosk.tytlabs.co.jp) \\ 4)-5) Virginia Tech - Wake Forest University Center for Injury Biomechanics \\ 443 ICTAS Building Stanger Street, Mail Code 0194 Blacksburg, VA 24061, USA
}

Received on June, 18,2010

Presented at the JSAE Annual Congress on May, 21,2010

\begin{abstract}
Over four thousand data sets of 6 DOF (degree of freedom) (3 linear +3 angular) accelerations measured at the head CG (center of gravity) were obtained from 19 college football players who voluntarily participated. Of these impacts, one hundred cases with high angular velocity were selected, and the set of 6 DOF head accelerations was applied to a detailed human head brain FE model. This study predicted the maximum $1^{\text {st }}$ principal strain and CSDM (Cumulative Strain Damage Measure) for each set and found significant correlations between CSDM and a proposed criterion with angular velocity and acceleration of the head CG.
\end{abstract}

KEY WORDS: (Standardized) Safety, Acceleration, Injury, Finite element method (FEM), (Free) Head, Brain [C1]

\section{Introduction}

Head injury constitutes $72.4 \%$ of all fatal cases considering primal injured body region based on police data of Japan ${ }^{(1)}$ in 2008. Although automotive related fatalities, which are number of victims who died within 30 days after accident, are continuously reduced to 6,023 , head injury is still remarkable problem in Japan. Considering accident patterns of those fatal cases, the police data also suggest that pedestrian $(29.4 \%)$ and cyclist $(29.3 \%)$ were more frequent than occupant $(21.3 \%)$. Feist et al. ${ }^{(2)}$ analyzed field data from a European database and found that pedestrians sustain head angular acceleration more frequently than linear acceleration in car-to-pedestrian accidents. The HIC (Head injury criterion) is widely used as an injury predictor of the head. However, due to the definition of HIC, it is difficult to find a correlation between HIC and angular head accelerations. Therefore, new head injury criteria for angular head impacts are needed.

Recently, a number of isolated human brain FE (finite element) models have been proposed and used to investigate the injury predictors. Most of those were validated against several series of PMHS (post mortem human subject) tests, and some researches proposed brain injury predictors based on FE models. However, due to limited number of experimental data, it is difficult to validate FE models in angular head accelerations well.

Besides PMHS tests, the high occurrence of concussions in football provides a unique opportunity to collect biomechanical data to characterize MTBI (mild traumatic brain injury). For example, Duma et al. ${ }^{(3)}$ presented a study to quantify head acceleration in collegiate football players by collecting over 3,000 impacts from 38 players using the HITS (Head Impact Telemetry
System: Simbex, Lebanon NH) measurement devices, in which one concussive event was measured. This HITS measurement device is capable of measuring resultant linear acceleration and impact location. However, it does not measure $\mathrm{x}, \mathrm{y}$, and $\mathrm{z}$ axis linear acceleration. Angular acceleration is also incapable of being measured. The six DOF (degree of freedom) measurement device (abbr. 6DOF sensor) improves the design of this measurement device to measure $\mathrm{x}, \mathrm{y}$, and $\mathrm{z}$ axis linear and angular accelerations ${ }^{(4)}$

The objectives of this study were 1) to obtain sets of three linear and three angular head accelerations using newly developed $6 \mathrm{DOF}$ sensors, 2) to apply the data to a well validated human brain FE model, and 3) to investigate correlations between FE model responses and head injury metrics.

\section{Methods}

\subsection{Measurement of head impact accelerations}

\subsubsection{Measurement device}

The 6DOF sensor, which utilizes twelve single-axis, high-g iMEMS accelerometers (ADXL193, Analog Devices, Norwood, MA), is designed to be integrated into Riddell Revolution football helmets. Twelve accelerometers are enclosed in the fabric padding, positioned in orthogonally oriented pairs at six different locations (Fig. 1). All accelerometers are orientated so that the sensing axis is tangential to the head's CG (center of gravity). The fabric pad also serves as a spring to keep the accelerometers in contact with the head (Fig. 2). When the helmet is impacted, the padding inside the helmet compresses and the helmet shifts 
the positions on the head. Therefore the measured accelerations are not helmet acceleration, but head acceleration ${ }^{(5)}$.

Since twelve accelerometers are redundant for the $6 \mathrm{DOF}$ measurement, an algorithm with an iterative optimization approach is used to solve for linear and angular acceleration ${ }^{(6)}$.
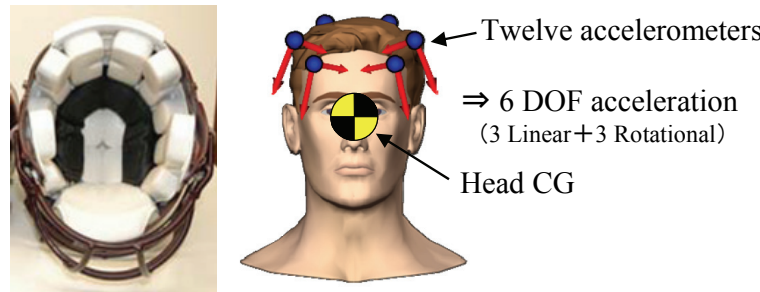

Fig. 1 6DOF sensor installed in a Riddell Revolution helmet (left) has 12 tangentially oriented accelerometers (right).
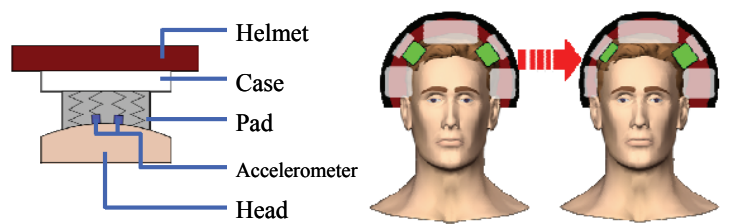

Fig. 2 Schematic of 6DOF sensor (left) and padding function which keeps accelerometers in contact with the head (right).

\subsubsection{Validation}

A total of 114 impact tests were conducted to assess the accuracy of the $6 \mathrm{DOF}$ sensor using an instrumented $50^{\text {th }}$ percentile male Hybrid III head and neck assembly by Rowson et al. (2010) ${ }^{(7)}$. The Hybrid III head was equipped with nine accelerometers (7264-2000B, Endevco, San Juan Capistrano, CA) in a 3-2-2-2 orientation; which allowed linear and angular acceleration to be calculated. The head and neck were mounted on a custom linear slide table built to NOCSAE (National Operating Committee on Standards for Athletic Equipment specification) ${ }^{(8)}$. Since the linear slide table permitted five degrees of freedom, head and neck orientation could be adjusted with high repeatability.

Efforts were made to simulate a helmet fitted to a human head while using the Hybrid III. This included inserting a lowfriction interface between the Hybrid III head and helmet ${ }^{(7)}$. This simulated helmet movement relative to the head that is seen in typical on-field impacts.

The helmeted Hybrid III head was struck with the pneumatic linear impactor with several combinations of impact velocities

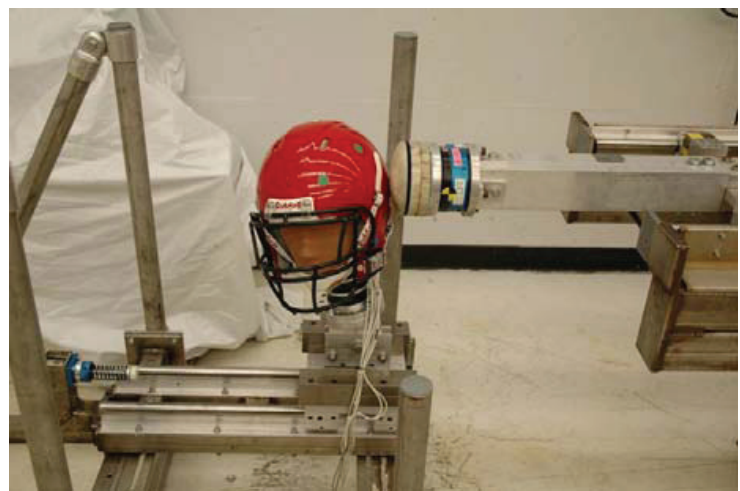

Fig. 3 Pneumatic linear impactor and helmeted Hybrid III head mounted on the linear slide table. and locations (Fig. 3). Impact velocities were chosen so that they would simulate a range of impact severities typically experienced in tackling and blocking. The impact velocities ranged from 3.0 to $9.0 \mathrm{~m} / \mathrm{s}$ and were based on the NFL (National Football League) reconstruction data ${ }^{(9)}$. To account for the various ways that a helmet can be struck, seven impact locations were chosen. Each combination was tested in four trials.

\subsubsection{Data acquisition system}

Data acquisition is triggered when any accelerometer exceeds $10 \mathrm{~g}$. The threshold of $10 \mathrm{~g}$ was decided based on a previous experimental study in which accelerations sustained by the head during daily activities were measured ${ }^{(10)}$. Data are collected for $40 \mathrm{~ms}$ at $1,000 \mathrm{~Hz}$, of which $8 \mathrm{~ms}$ are pre-trigger and $32 \mathrm{~ms}$ are post-trigger. After each impact is recorded, the data are sent to a computer on the sideline via a 903-927 MHz wireless transceiver. For each impact, linear and angular accelerations for each axis and impact location are measured.

\subsubsection{Data collection}

The 6DOF sensors were installed in the helmets of 19 Virginia Tech football players throughout the 2007 and 2008 Virginia Tech college football seasons. All 19 instrumented players were either offensive or defensive linemen. Each player that participated in the study gave written informed consent with Institutional Review Board approval from both Virginia Tech and the Edward Via College of Osteopathic Medicine. Linear and angular accelerations were recorded for every impact instrumented players experienced during games and practices.

All data were up-sampled from $1,000 \mathrm{~Hz}$ to $10,000 \mathrm{~Hz}$ by linear interpolation. The data were then filtered at CFC (Channel Frequency Class) 180.

\subsection{Human head brain FE analysis}

An isolated human head brain FE model was used for this study (Fig. 4). The model consisted of 49,579 elements (24,096 solid, 25,119 shell and 364 seatbelt elements) had a mass of 4.39 $\mathrm{kg}$ and an appropriate position for the CG. Those inertia data of the head model were determined based on the anthropometry of AM50 (11). The skull model consisted of frontal, parietal, temporal, occipital, sphenoid, ethmoid, lacrimal, nasal, vomer, zygomatic, maxilla, mandible, and palatine bones. The skull sutures, which connected the cranial bones, were modeled with solid elements. The brain model consists of all hexagonal solid elements representing the cerebrum, cerebellum, brainstem with distinct white and gray matter, and CSF (cerebral spinal fluid). Additionally, solid elements were used to represent the sagittal

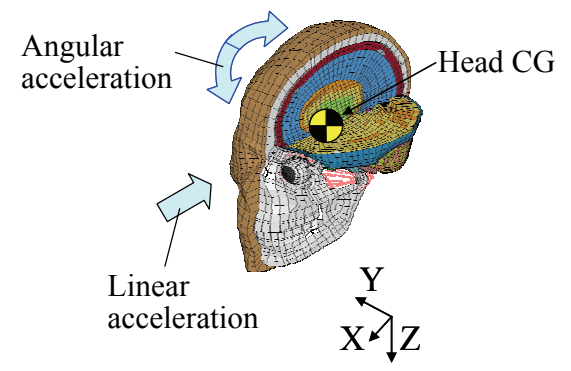

Fig. 4 Human head brain FE model and location of CG. 
sinus, and shell elements were used to represent the dura, pia, arachnoid, meninx, falx cerebri, and tentorium.

The model was already validated against a series of linear head impact ${ }^{(12)}$ and two series of angular impacts ${ }^{(13,14)}$ (Fig. 5), and presented high bio-fidelity. Solid and gray lines represent the experimental data and simulation results, respectively. The origin is the CG of the head. The orientation of the head and the direction of angular acceleration are illustrated inside of the graph. Further details about this model are written in a previous publication $^{(15)}$.

It was hypothesized that the skull deformation caused by head impacts below the threshold for fracture does not affect the brain motion. Since none of the head acceleration cases used for

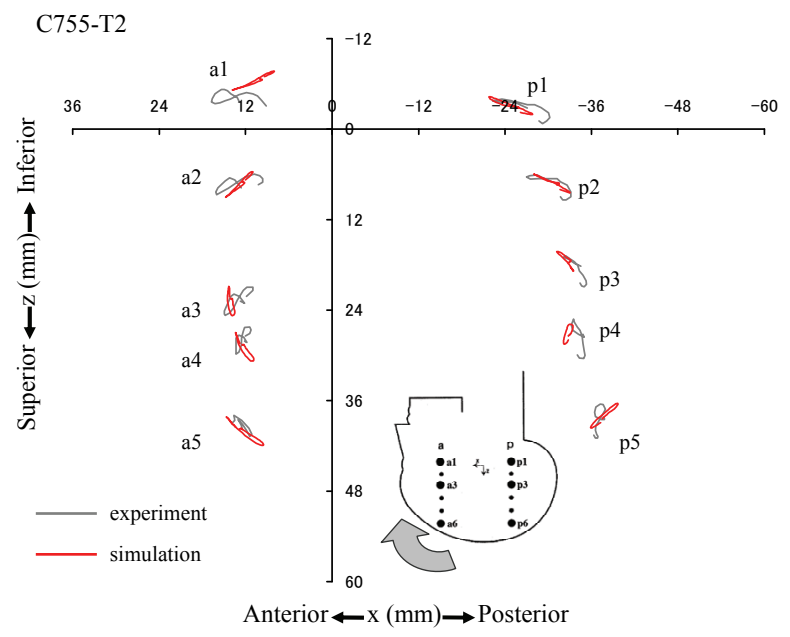

Fig. 5 Brain motions at various locations in a sagittal plane during Test C755-T2 ${ }^{(14)}$.

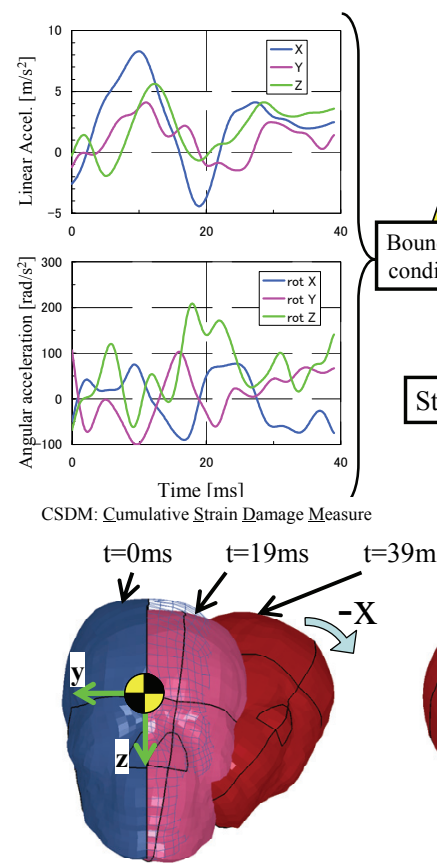

Front view

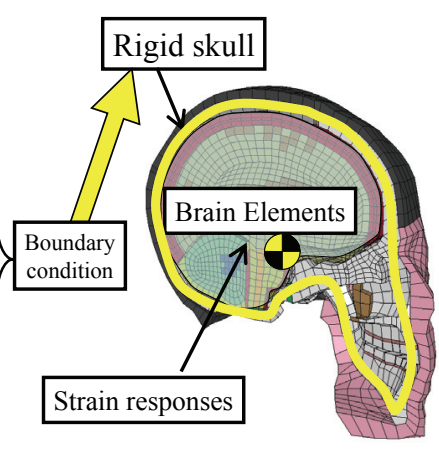

THUMS Brain Model

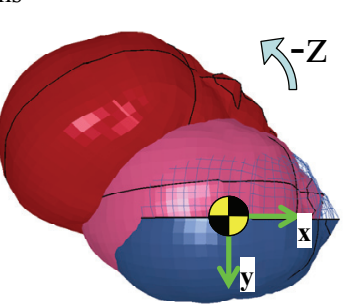

Top view

Fig. 6 FE analysis methods: 6 DOF head accelerations were applied to a rigid skull model (upper). The resulting motion was described with respect to the local coordinate system for the model (lower). this study presented bone fracture, the skull of the model was assumed to be rigid. By assuming this, the measured 6DOF accelerations could be directly input as boundary conditions of the head through the skull (Fig. 6).

All simulations were conducted by a commercially available FE solver, LS-DYNA 971 Rev. 2, SMP (Shared Memory Parallel) version (LSTC, Livermore, CA) using only single core of an Intel Xeon 64 bit based computer running on a Linux operating system.

\subsection{Injury metrics}

Nine injury metrics were calculated and analyzed in this study: maximum first principal strain (FPS), Cumulative Strain Damage Measure (16) (CSDM), HIC 15 , maximum linear velocity and acceleration, maximum angular velocity and acceleration, Head Injury Power ${ }^{(17)}$ (HIP), and Power Rotational Head Injury Criterion $\left(\mathrm{PRHIC}_{36}\right.$ ). The latter is a newly proposed criterion based on angular velocities and accelerations of the head CG. Details of CSDM and PRHIC are described in the following.

\subsubsection{CSDM}

Takhounts et al. ${ }^{(16)}$ proposed CSDM for evaluating DAI (Diffused Axonal Injury). This criterion defines brain damage for overstretched elements in FE model. Once an element experiences maximum FPS over a threshold, the element was assumed as damaged. The damaged elements were accumulated and the total volume of those was counted. The CSDM shows the volume percentages of damaged elements in whole cerebrum, so that the threshold of DAI is determined by values of CSDM (Fig. 7). When the threshold of maximum FPS was $15 \%$, the variable term was written as "CSDM $15 \%$ " in this study.

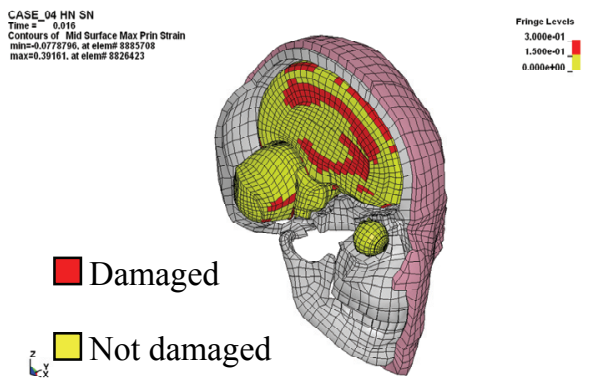

Fig. 7 A sample of strain distribution in human head brain FE model. Elements which experienced over $15 \%$ of FPS were assumed as damaged.

\subsubsection{PRHIC}

Severity index (SI) was proposed by Gadd (1966) ${ }^{(17)}$ and was a precursor to HIC. The severity index was designed to have strong agreement with the Wayne State University Tolerance Curve (WSUTC: Patrick et al., $1963^{(18)}$ ) and is shown in equation (1).

$$
S I=\int a^{n} d t \leq 1,000
$$

where $a$ is either of acceleration, force, or pressure, which is a response function producing threshold of injury, and $n$ is weighting factor equal to 2.5.

Versace (1971) ${ }^{(19)}$ advanced upon SI and developed HIC, which is the current injury metric for head injury used in the 
FMVSS 208 standard. The equation is represented as equation (2).

$$
H I C=\left[\left\{\frac{1}{\left(t_{2}-t_{1}\right)} \int_{t_{1}}^{t_{2}} a(t) d t\right\}^{2.5}\left(t_{2}-t_{1}\right)\right]_{\max }
$$

where $a(t)$ is resultant linear acceleration, and $t_{1}$ and $t_{2}$ represent the initial and final integral times which HIC is calculated over $\left(t_{1}\right.$ and $t_{2}$ are selected to maximize HIC). Determining HIC involves a computational solver which seeks the maximum value of HIC over a portion of the pulse. The maximum time duration was set as $36 \mathrm{msec}$ at first, however current standards use $15 \mathrm{msec}$. HIC is good injury metric for skull fracture ${ }^{(20)}$.

Since the SI and HIC equations are mostly based on the WSUTC, the variables typically only consist of resultant linear acceleration; although Gadd did not limit the variations of variables.

Newman et al. ${ }^{(21)}$ described HIP (Head Injury Power) as a power expression of the human head when the kinematics of the head assumed as a rigid motion.

$$
H I P=\sum m \cdot a_{i} \cdot \int a_{i} d t+\sum I_{i i} \cdot \alpha_{i} \cdot \int \alpha_{i} d t
$$

where $m$ is mass of the head $(\mathrm{kg}), a_{i}$ is linear acceleration $\left(\mathrm{m} / \mathrm{s}^{2}\right)$, $I_{i i}$ is MOI (moment of inertia) $\left(\mathrm{kg} \cdot \mathrm{m}^{2}\right)$, and $\alpha_{i}$ is angular acceleration $\left(\mathrm{rad} / \mathrm{s}^{2}\right)$. Coefficient of mass is $4.5 \mathrm{~kg}$, and those of MOI for $\mathrm{x}, \mathrm{y}$, and $\mathrm{z}$ directions are $0.016,0.024$, and $0.022 \mathrm{~kg} \cdot \mathrm{m}^{2}$, respectively.

The equation (3) includes both of linear and angular power terms. However, this study focuses on angular acceleration, so that linear term was omitted from consideration. Therefore, the angular HIP_ang $(t)$ represents the rate of change in angular head kinetic energy, which is described as equation (4).

$$
\begin{aligned}
H I P_{-} \operatorname{ang}(t) & =\sum I_{i i} \cdot \alpha_{i} \cdot \int \alpha_{i} d t \\
& =0.016 \cdot \alpha_{x} \cdot \int \alpha_{x} d t+0.024 \cdot \alpha_{y} \cdot \int \alpha_{y} d t \\
& +0.022 \cdot \alpha_{z} \cdot \int \alpha_{z} d t
\end{aligned}
$$

The plots of maximum value of HIP_ang $(t)$ versus the time duration of HIP_ang formulate similar distributions as SI or HIC definitions. This finding is similar to previous work ${ }^{(22)}$ of investigating angular kinematics using animal models. Therefore, this study substituted $H I P \_a n g(t)$ for resultant linear acceleration of $a(t)$ in the equation (1), and proposed a new injury metric: the PRHIC (Power Rotational Head Injury Criterion) as equation (5). For SI or HIC definitions, the power 2.5 was determined from tolerance curve using both injurious and non-injurious data plots. However, it is currently impossible to determine new power value for PRHIC since 6DOF sensor data did not have any concussive data. Therefore we assumed the power 2.5 for PRHIC as well.

$$
\text { PRHIC }=\left[\left\{\frac{1}{\left(t_{2}-t_{1}\right)} \int_{t_{1}}^{t_{2}} H I P_{-} \text {ang }(t) d t\right\}^{2.5}\left(t_{2}-t_{1}\right)\right]_{\max }
$$

An ultimate goal of this research is applying new injury criterion to automotive safety. Since HIC used 15 and 36 time durations for automotive safety standards in past history, we adopted same time durations into injury criteria of the head. NFL study reported that football impact has 15 to $20 \mathrm{msec}$ time duration for the acceleration peaks ${ }^{(23)}$. In addition, since brain elements consist of viscous solid materials, peak of brain responses such as the first principal strain delays approximately $15 \mathrm{msec}$ from that of angular acceleration. Therefore, time duration for PRHIC which is a brain injury predictor was selected as $36 \mathrm{msec}$.

\section{Results}

\subsection{Validation for $6 \mathrm{DOF}$ sensor}

The linear relationship for resultant linear acceleration between the 6DOF sensor and Hybrid III data correlated strongly $\left(\mathrm{R}^{2}=0.88\right)$. Correlation of resultant angular acceleration was also significant $\left(R^{2}=0.85\right)$. Error was calculated by dividing the difference in acceleration between the 6DOF sensor and the Hybrid III, by the Hybrid III acceleration. The average error between the 6DOF sensor and Hybrid III for the resultant linear accelerations was $1 \%$. That for the resultant angular accelerations was $3 \%$. Error was not significantly affected by impact location and direction ${ }^{(7)}$
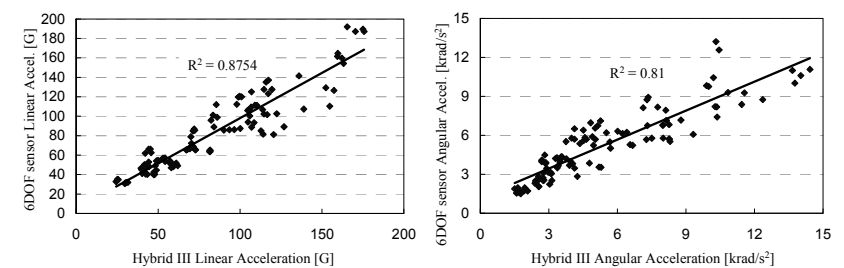

Fig. 8 Linear relationship between 6DOF sensor and Hybrid III in peak resultant linear (left) and resultant angular (right) acceleration.

Referring other human subject testing devices and techniques, the NFL video analysis ${ }^{(24)}$ was reported to have error as high as $15 \%$; while the original HITS technology has an error of $8 \pm 11 \%$ (mean $\pm \mathrm{SD})^{(25)}$. Considering the vast amounts of data to be collected with the 6DOF sensor and the error levels of other accepted experiments, the inherent error of the $6 \mathrm{DOF}$ sensor is acceptable.

\subsection{Head impact data collection from $6 \mathrm{DOF}$ sensor}

A total of 4,709 impacts were recorded during practices and games for the 19 instrumented players during the 2007 and 2008 Virginia Tech football seasons. No instrumented player sustained MTBI in this study. Table 1 displays the frequency of impacts over specified resultant acceleration thresholds for linear and angular acceleration. For resultant linear acceleration, the majority of the impacts were under $20 \mathrm{~g}$ in severity. Of the 4,709 impacts, 38 were greater than $80 \mathrm{~g}$, which is the nominal injury value derived by the NFL study. For resultant angular acceleration, roughly half of the impacts were less than 1,000 $\mathrm{rad} / \mathrm{s}^{2}$ in severity. Only 30 were greater than $6,000 \mathrm{rad} / \mathrm{s}^{2}$, which 
is the nominal injury value derived in the NFL study. These data are described in detail by Rowson et al. ${ }^{(4)}$.

Table 1 Frequency of impacts above specified resultant acceleration thresholds

\begin{tabular}{|c|c|c|c|c|c|}
\hline $\begin{array}{c}\text { Linear } \\
\text { acceleration }\end{array}$ & 2007 & 2008 & $\begin{array}{c}\text { Angular } \\
\text { acceleration }\end{array}$ & 2007 & 2008 \\
\hline$>0 \mathrm{~g}$ & 1712 & 2997 & $>0 \mathrm{rad} / \mathrm{s}^{2}$ & 1712 & 2997 \\
\hline$>20 \mathrm{~g}$ & 684 & 1128 & $>1000 \mathrm{rad} / \mathrm{s}^{2}$ & 875 & 1303 \\
\hline$>40 \mathrm{~g}$ & 172 & 259 & $>2000 \mathrm{rad} / \mathrm{s}^{2}$ & 339 & 404 \\
\hline$>60 \mathrm{~g}$ & 52 & 70 & $>3000 \mathrm{rad} / \mathrm{s}^{2}$ & 143 & 141 \\
\hline$>80 \mathrm{~g}$ & 11 & 27 & $>4000 \mathrm{rad} / \mathrm{s}^{2}$ & 57 & 59 \\
\hline$>100 \mathrm{~g}$ & 3 & 14 & $>5000 \mathrm{rad} / \mathrm{s}^{2}$ & 23 & 33 \\
\hline$>120 \mathrm{~g}$ & 1 & 9 & $>6000 \mathrm{rad} / \mathrm{s}^{2}$ & 12 & 18 \\
\hline$>140 \mathrm{~g}$ & 0 & 2 & $>7000 \mathrm{rad} / \mathrm{s}^{2}$ & 5 & 12 \\
\hline$>160 \mathrm{~g}$ & 0 & 0 & $>8000 \mathrm{rad} / \mathrm{s}^{2}$ & 4 & 4 \\
\hline$>180 \mathrm{~g}$ & 0 & 0 & $>9000 \mathrm{rad} / \mathrm{s}^{2}$ & 1 & 1 \\
\hline
\end{tabular}

Although moderate levels of both linear and angular forms of acceleration combined can often cause severe brain injuries ${ }^{(26)}$, it is generally believed that concussion mechanisms relate head impact severities and magnitudes of brain responses in impact biomechanics community. For example, NFL research group selected only severe impact events from numerious head impacts to reconstruct football head impacts ${ }^{(9)}$. Therefore this study also focused on impact severities of individual impacts without any considerations of numbers of head impacts or those accumulations which individual players earn during one-day football activity.

Margulies and Thibault ${ }^{(27)}$ described injury probability and thresholds for DAI on a graph of angular acceleration versus angular velocity. In the same manner, Fig. 9 shows data plots of $6 \mathrm{DOF}$ sensors with the thresholds, which are retrospectively obtained from literature data ${ }^{(22,28-29)}$, on the angular acceleration vs. angular velocity graph. Although 6DOF dataset does not contain any injurious cases, some cases were greater than the thresholds. In order to investigate the strong angular head impact

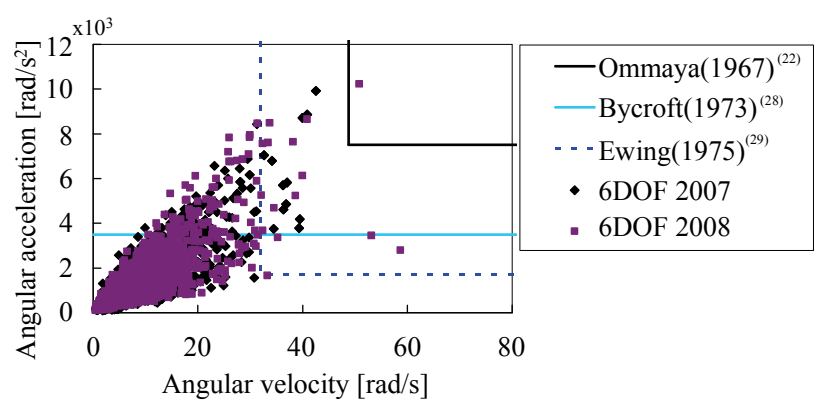

Fig. 9 Angular acceleration vs. angular velocity of 6DOF sensor data with threshold lines obtained from literatures ${ }^{(22,28-29)}$. data, this study selected fifty cases with high angular velocity from each of 2007 and 2008 datasets, and the total 100 sets of 6 DOF head accelerations were applied to a human head brain FE model.

\subsection{Human head brain FE model analysis}

The 6DOF acceleration data were used to calculate head injury metrics of $\mathrm{HIC}_{15}$, maximum linear velocity and acceleration, maximum angular velocity and acceleration, HIP, and $\mathrm{PRHIC}_{36}$. In addition, FE analysis using the human head brain FE model presented brain strain outcomes of maximum FPS and CSDM $10 \%, 15 \%$, and $20 \%$. Each FE analysis for $40 \mathrm{~ms}$ termination times took about four hours on a single core Xeon 3.0GHz CPU machine.

Table 2 shows correlations between variables of head injury metrics and results of $\mathrm{FE}$ analysis. The coefficient of determination $\left(\mathrm{R}^{2}\right)$ for $\mathrm{HIC}_{15}$ was less than 0.11 against $\mathrm{FE}$ strain variables of cerebrum (Fig. 10). Similarly, maximum linear velocity, linear acceleration, angular velocity and HIP did not show any correlation with FE results. The maximum angular acceleration was somewhat correlated $\left(\mathrm{R}^{2}=0.69\right)$ with CSDM $10 \%$. On the contrary, PRHIC $_{36}$, which this study newly proposed, was correlated well with CSDM values (Fig. 10). Specially, the correlation with CSDM $20 \%$ was significant $\left(\mathrm{R}^{2}=0.84\right)$.

\section{Discussion}

\subsection{Correlations with CSDM}

The values of FE strain variables of cerebrum elements did not show any correlations against HIC, linear velocity, linear acceleration, and HIP. The HIC, which is defined by integrated linear acceleration as equation (1), is believed as an injury predictor for skull fractures. The HIP definition includes not only angular accelerations but also linear acceleration terms (equation (2)). Therefore, linear acceleration can be believed as insensitive to maximum FPS or CSDM of the cerebrum.

On the contrary, angular acceleration shows stronger correlations with FPS and CSDM than linear acceleration. Definition of PRHIC contains both of angular velocity and acceleration, so that the correlations with $\mathrm{FE}$ variables would be strong. Therefore, this study can suggest that two injury predictors are needed for head injury: one is based on linear accelerations for skull fracture, and another is based on angular

Table 2 Coefficient of determinations ( $\mathrm{R}^{2}$, upper) and $p$-values (lower) between FE strain variables and head injury metrics.

\begin{tabular}{c|ccccccc}
\hline $\mathbf{N}=\mathbf{1 0 0}$ & $\mathbf{H I C}_{\mathbf{1 5}}$ & $\begin{array}{c}\text { Max. linear } \\
\text { velocity }\end{array}$ & $\begin{array}{c}\text { Max. linear } \\
\text { acceleration }\end{array}$ & $\begin{array}{c}\text { Max. angular } \\
\text { velocity }\end{array}$ & $\begin{array}{c}\text { Max. angular } \\
\text { acceleration }\end{array}$ & HIP & PRHIC $_{\mathbf{3 6}}$ \\
\hline \multirow{2}{*}{ Max FPS } & 0.11 & 0.11 & 0.20 & 0.23 & 0.30 & 0.25 & 0.25 \\
& $p=0.0009$ & $p=0.0004$ & $p=4.0 e-6$ & $p=4.7 e-7$ & $p=4.1 e-9$ & $p=1.5 e-7$ & $p=1.4 e-7$ \\
\hline CSDM & 0.04 & 0.001 & 0.10 & 0.25 & 0.69 & 0.18 & 0.64 \\
$10 \%$ & $p=0.059$ & $p=0.67$ & $p=0.002$ & $p=1.5 e-7$ & $p=5.8 e-27$ & $p=9.3 e-6$ & $p=3.1 e-23$ \\
\hline CSDM & 0.01 & 0.003 & 0.06 & 0.28 & 0.41 & 0.12 & 0.75 \\
$15 \%$ & $p=0.24$ & $p=0.59$ & $p=0.018$ & $p=2.0 e-8$ & $p=7.9 e-13$ & $p=0.0003$ & $p=0.00$ \\
\hline CSDM & 0.02 & 0.008 & 0.06 & 0.27 & 0.32 & 0.14 & 0.84 \\
$20 \%$ & $p=0.15$ & $p=0.38$ & $p=0.011$ & $p=3.1 e-8$ & $p=1.0 e-9$ & $p=0.0002$ & $p=0.00$ \\
\hline
\end{tabular}



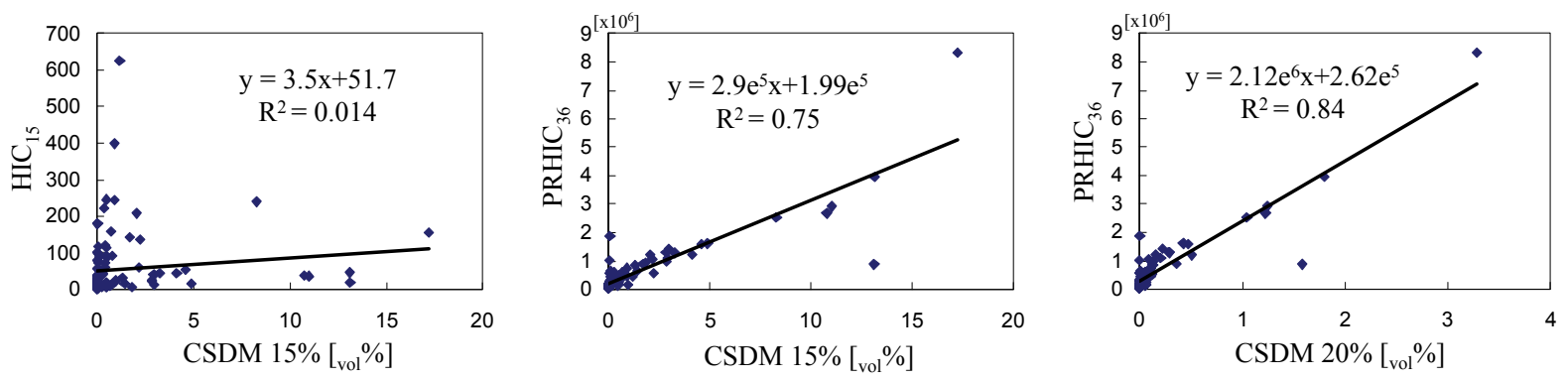

Fig. 10 Injury metrics of $\mathrm{HIC}_{15}$ and $\mathrm{PRHIC}_{36}$ vs. CSDM 15\% and CSDM 20\% obtained from FE analysis.

accelerations for brain injury.

The CSDM was proposed as a predictor of Diffuse Axonal Injuries (DAI) by using brain FE models ${ }^{(16)}$. In such injuries, the damaged tissue is expected to be spread throughout a large volume in the cerebrum. In FE analysis using CSDM, the smaller the First Principal Strain (FPS) level at which an element is considered damaged, the higher the predicted volume of damaged brain elements becomes. For example, in this study, a FPS threshold of $15 \%$ strain, resulted in a $17.2 \%$ of damaged brain elements. When the FPS threshold was set to $20 \%$, only $3.2 \%$ of the elements reached the level of damaged (See maximum abscissa values in Fig. 10). However, an important observation was done when analyzing the statistical correlation of CSDM with other injury predictors. For high strain threshold (20\%), the strongest correlation between CSDM and PRHIC was found $\left(\mathrm{R}^{2}=0.84\right)$. For such CSDM values, the volume of damaged elements is small. Since Traumatic Brain Injuries sustained by football players are usually milder than DAI, a further verification for strain thresholds is required to define MTB injuries.

\subsection{Limitation}

This study obtained over four thousand impact data using 6DOF sensors. However, it is impossible to earn a threshold for brain injury using only 6DOF dataset because concussion cases were not included. In addition, $6 \mathrm{DOF}$ football data did not have skull fracture, while automotive crashes would contain skull fractures in head injury. Therefore, further verification studies are needed for CSDM and PRHIC using other head impact data including skull fractures.

\section{Conclusions}

Following four specific conclusions can be drawn for the investigation of brain response simulations for angular head impacts using a human brain FE model.

- The $6 \mathrm{DOF}$ sensor showed a strong linear correlation when validated against head acceleration of a Hybrid III dummy.

- Total 4,709 impacts were collected by instrumenting Virginia Tech football players' helmets with the 6DOF sensor through the 2007 and 2008 football seasons.

- PRHIC $_{36}$ which is calculated from integrated angular acceleration and angular velocity was proposed in this study.
- Although 6DOF data did not contain any concussion cases, PRHIC $_{36}$ presented strong correlation with CSDM $20 \%$.

\section{References}

(1) Cabinet Office, Government of Japan: White Paper on Traffic Safety in Japan 2009. Nikkei Insatsu, pp.158-160, Tokyo, (2009) (2) Feist F, Gugler J, Arregui-Dalmases C, Del Pozo de Dios E, Lopez-Valdèz F, Deck C, and Willinger R: Pedestrian Collisions with flat fronted vehicles: Injury patterns and importance of rotational accelerations as a predictor for traumatic brain injury (TBI). $21^{\text {st }}$ International Technical Conference on the Enhanced Safety of Vehicles (ESV), pp.15-18, Stuttgart, Germany, (2009)

(3) Duma SM, Manoogian SJ, Bussone WR, Brolinson PG, Goforth MW, Donnenwerth JJ, Greenwald RM, Chu JJ and Crisco JJ: Analysis of real-time head accelerations in collegiate football players. Clin J Sport Med, 15(1), pp.3-8, (2005)

(4) Rowson S, Brolinson G, Goforth M, Dietter D, and Duma S: Linear and Angular Head Acceleration Measurements in Collegiate Football. Journal of Biomechanical Engineering, Vol.131(6): 061016, (2009)

(5) Manoogian S, McNeely D, Duma S, Brolinson G and Greenwald R: Head acceleration is less than 10 percent of helmet acceleration in football impacts. Biomed Sci Instrum, 42: pp.383388, (2006)

(6) Chu JJ, Beckwith JG, Crisco JJ, and Greenwald R: A Novel Algorithm to Measure Linear and Rotational Head Acceleration Using Single-Axis Accelerometers. J. Biomech., 42 (Suppl. 1), p. S534, (2006).

(7) Rowson S, Beckwith J, Chu J, Leonard D, Greenwald R, and Duma S: A six degree of freedom head acceleration measurement device for use in football. Journal of Applied Biomechanics, (2010), In Press.

(8) National Operating Committee on Standards for Athletic Equipment: Standard linear impactor test method and equipment used in evaluating the performance characteristics of protective headgear and face guards. NOCSAE DOC (ND) 081- 04m04, (2006)

(9) Pellman EJ, Viano DC, Tucker AM, Casson IR and Waeckerle JF: Concussion in professional football: reconstruction of game impacts and injuries. Neurosurgery, 53(4): pp.799-812; discussion pp.812-814, (2003)

(10) Ng TP, Bussone WR, Duma SM: The effect of gender and body size on linear accelerations of the head observed during daily activities. Biomed Sci Instrum; 42:25-30. (2006)

(11) Schneider LW, Robbins DH, Pflug MA, and Snyder RG: Development of anthropometrically based design specifications for an advanced adult anthropomorphic dummy family, Volume 1. UMTRI-83-53-1, NHTSA, (1983) 
(12) Nahum AM, Smith R, and Ward CC: Intracranial pressure dynamics during head impact. Proc. of the 21st Stapp Car Crash Conference, SAE Paper No.770922, pp.339-366, (1977)

(13) Trosseille X, Tarriere C, Lavaste F, Guillon F, and Domont A: Development of a F.E.M. of the human head according to a specific test protocol. Proc. of 36th Stapp Car Crash Conference, SAE Paper No.922527, (1992)

(14) Hardy WN, Foster CD, Mason MJ, Yang KH, King AI, and Tashman S: Investigation of head injury mechanisms using neutral density technology and high-speed biplanar X-ray. Stapp Car Crash Journal, vol.45, SAE paper No.2001-22-0016, pp.337$368,(2001)$

(15) Kimpara H, Nakahira Y, Iwamoto M, Miki K, Ichihara K, Kawano S, and Taguchi T: Investigation of Anteroposterior HeadNeck Responses during Severe Frontal Impacts Using a BrainSpinal Cord Complex FE Model. Stapp Car Crash Journal, 50, pp.509-544, (2006)

(16) Takhounts EG, Crandall JR, Darvish K: On the Development of the SIMon Finit Element Head Model. Stapp Car Crash Journal, vol.47, pp.107-133, (2003)

(17) Gadd CW: Use of a weighted-impulse criterion for estimating injury hazard. Proc. of $10^{\text {th }}$ Stapp Car Crash Conference, SAE Paper 660793, pp.164-174, (1966)

(18) Patrick LM, Lissner HR, Gurdjian ES: Survival by design head protection. Proc. of $7^{\text {th }}$ Stapp Car Crash Conference, SAE Paper 1963-12-0036: pp.483-499, (1963)

(19) Versace J: A review of the severity index. Proc. of the $15^{\text {th }}$ Stapp Car Crash Conference, SAE Paper 710881, pp.771-796, (1971)

(20) Hertz E: A note on the head injury criterion (HIC) as a predictor of the risk of skull fracture. $37^{\text {th }}$ Annual Proceedings of the AAAM., pp.303-312, (1993)

(21) Newman JA, Shewchenko N, and Welbourne E: A proposal new biomechanical head injury assessment function - the maximum power index. Stapp Car Crash Journal, 44, 2000-01SC16, pp.215-247, (2000)

(22) Ommaya AK, Yarnell P, Hirsch A, Harris D: Scaling of experimental data on cerebral concussion in sub-human primates to concussive thresholds in man. Proc. $11^{\text {th }}$ Stapp Car Crash Conf, SAE Paper No.670906, (1967)

(23) Viano DC, Casson IR, Pellman EJ, Zhang L, King AI, Yang $\mathrm{KH}$ : Concussion in professional football: brain responses by finite element analysis: part 9. Neurosurgery, 57(5):891-916, (2005)

(24) Pellman EJ, Viano DC, Tucker AM and Casson IR: Concussion in professional football: location and direction of helmet impacts-Part 2. Neurosurgery, 53(6): pp.1328-1340; discussion pp.1340-1341, (2003)

(25) Funk JR, Duma SM, Manoogian SJ and Rowson S: Biomechanical risk estimates for mild traumatic brain injury. Annu Proc Assoc Adv Automot Med, 51: pp.343-361, (2007)

(26) King AI, Yang KH, Zhang L and Hardy W: Is Head Injury Caused by Linear or Angular Acceleration? Proceedings of the International Research Conference on the Biomechanics of Impacts (IRCOBI), Lisbon, (2003)

(27) Margulies SS and Thibault LE: A Proposed Tolerance Criterion for Diffuse Axonal Injury in Man. Journal of Biomechanics, vol.25, No.8, pp.917-923, (1992)

(28) Bycroft GN: Mathematical model of a head subjected to an angular acceleration. Journal of Biomechanics, vol.6, pp.487-495, (1973)

(29) Ewing C, Thomas D, Lustic L, Becker E, Willems G, Muzzy W: The effect of the iitial position of the head and neck on the dynamic response of the human head and neck to $-\mathrm{Gx}$ impact acceleration. Proc. $19^{\text {th }}$ Stapp Car Crash Conference, SAE Paper 751157, pp.487-512, (1975) 